\title{
Unconscious semantic priming extends to novel unseen stimuli
}

\author{
Lionel Naccache*, Stanislas Dehaene \\ Unité INSERM 334, Service Hospitalier Frédéric Joliot, CEA/DRM/DSV, 4, Place du Général Leclerc, \\ 91401 Orsay Cedex, France
}

Received 13 May 2000; received in revised form 23 October 2000; accepted 7 November 2000

\begin{abstract}
Many subliminal priming experiments are thought to demonstrate unconscious access to semantics. However, most of them can be reinterpreted in a non-semantic framework that supposes only that subjects learn to map non-semantic visual features of the subliminal stimuli onto motor responses. In order to clarify this issue, we engaged subjects in a number comparison task in which the target number was preceded by another invisible masked number. We show that unconscious semantic priming occurs even for prime stimuli that are never presented as target stimuli, and for which no stimulus-response learning could conceivably occur. We also report analyses of the impact of the numerical relation between prime and target, and of the impact of learning on priming, all of which confirm that unconscious utilization of semantic information is indeed possible. (C) 2001 Elsevier Science B.V. All rights reserved.
\end{abstract}

Keywords: Unconscious; Semantic priming; Novel unseen stimuli

\section{Introduction}

A debated issue in cognitive neuroscience concerns the existence of an unconscious semantic processing of masked primes. There are many convincing demonstrations that a masked visual stimulus, which is presented too briefly to be consciously seen, can nevertheless be processed at various sub-semantic levels. Recent examples include visual word identification (Ferrand, Grainger, \& Segui,

* Corresponding author. Tel.: +33-1-69-86-78-40; fax: +33-1-69-86-78-16.

E-mail address: naccache@shfj.cea.fr (L. Naccache). 
1994; Forster, 1999), processing of facial emotions (Whalen et al., 1998), and stimulus-response associations (Eimer \& Schlaghecken, 1998; Neumann \& Klotz, 1994). Several demonstrations of unconscious access to the meaning of masked primes have also been put forward (Draine \& Greenwald, 1998; Dell'Acqua \& Grainger, 1999; Greenwald, 1996; Luck, Vogel, \& Shapiro, 1996; Marcel, 1983).

Most of this literature focuses exclusively on the existence of unconscious access to semantics, leaving open the more general scientific issue of whether semantic manipulations can be performed unconsciously. Can the unconscious meaning of a word be mentally manipulated and used to bias behavior? We recently addressed this issue using a number comparison task with masked primes (Dehaene et al., 1998). Subjects pressed a right or left key to decide whether a visually presented target number was larger or smaller than 5. Unbeknownst to them, another number was presented for $43 \mathrm{~ms}$ just before the target, surrounded by masks that made it invisible. Behavioral and brain imaging (ERPs and fMRI) measures revealed that this numerical prime nevertheless yielded a covert motor interference effect. Subjects were faster when the prime and target numbers fell on the same side of 5 , and therefore called for the same motor response (congruent trials), than when they did not (incongruent trials). Furthermore, measures of motor activity showed directly that subjects were programming a covert motor response appropriate to the prime before implementing the overt motor response appropriate to the target. We concluded that information extracted from the unseen prime was processed through an entire series of processing stages that included the semantic categorization of the numerical prime as larger or smaller than 5 , and that proceeded all the way down to a motor level.

Recently, however, an alternative non-semantic interpretation of masked priming experiments has been gaining strength (Abrams \& Greenwald, in press; Damian, in press; Eimer \& Schlaghecken, 1998; Neumann \& Klotz, 1994). Abrams and Greenwald asked subjects to evaluate the valence of consciously seen targets words as positive or negative. The prior presentation of a masked word, whose valence could be congruent or incongruent with the upcoming target, was shown to facilitate or interfere with the subjects' response. This seemed to prove that the masked prime was categorized semantically as positive or negative. Crucially, however, Abrams and Greenwald went on to demonstrate that the priming effect was entirely due to the fact that the prime words were also presented as conscious targets in other trials. When they examined generalization to novel primes that were never seen consciously, priming was obtained only inasmuch as some of their letter fragments matched those of a word from the target list. For instance, after repeated conscious classification of the words smut and bile as negative, the subliminal prime smile primed the negative response, not the positive one. This result suggests that the priming effect, in this situation, was not due to a subliminal access to semantics. Rather, subjects had learned to respond rapidly to fragments of the target strings with specific left or right key presses, and this sensorimotor learning generalized to other primes made of the same fragments. A similar conclusion has recently been reached by Damian on the basis of a lack of generalization of subliminal priming to novel primes in a semantic categorization task. Those results can be captured by the 
Neumann and Klotz (1994) direct motor specification hypothesis (hereafter DMS), according to which subjects unconsciously associate each visual stimulus with the adequate response, thus bypassing semantic access.

Could it be the case that under conditions of unconscious perception, the only available pathway is such a non-semantic associative response chain? Our number priming results (Dehaene et al., 1998) can certainly be reinterpreted non-semantically within the DMS framework. Rote stimulus-response learning was possible in our experiment because only eight stimuli (the numbers 1, 4, 6 and 9 in Arabic or in verbal notation) were used repeatedly. Furthermore, and crucially, the same numerical stimuli were used as both primes and targets. Although the instructions were changed in the middle of the experiments, subjects could have rapidly learned to associate each visual stimulus (or a fragment of it) with the corresponding motor response. Following this learning period, the response priming effect we observed could have arisen entirely from a sub-semantic visuomotor association process.

Because the DMS framework seems potentially capable of explaining away most, if not all, previous experiments thought to demonstrate unconscious semantic priming, it becomes crucial to obtain direct evidence for or against it. The purpose of the present paper is to provide such evidence by examining whether the numerical priming effect generalizes to novel unseen primes. We replicate our number priming experiment with two sets of numerical primes. Some are number words or Arabic numerals that can also appear as conscious targets (old set primes). Others are novel numbers that never appear as targets, and thus are never consciously perceived during the entire experiment (new set primes). The semantic and DMS hypotheses make contrasting predictions as to whether those primes should cause response priming. If the DMS hypothesis is correct, response priming should be obtained with the old set primes, but it should not be significant with the new set primes because the subjects have had no occasion to associate their visual shapes with a specific motor response. If, however, the semantic interpretation is correct and the response priming effect reflects a genuine unconscious categorization of the primes at the semantic level, then this effect should extend to the new set primes. Subjects should unconsciously apply the semantic number comparison task to all the numerical primes. This should result in an interference effect for all primes, whether new or old.

\section{Experiment 1}

\subsection{Materials and methods}

\subsubsection{Subjects}

Eighteen right-handed undergraduate students (nine males) ranging in age from 18 to 31 years (mean age 21.4 years, median age 21 years) volunteered for this study. All of them were naive as to the purpose of the experiment, gave their informed consent and received $30 \mathrm{FF}$ (4.6 Euros). 


\subsubsection{Instructions}

Subjects were told that they would see a target number between 1 and 9 , excluding 5 , and that they would have to compare it to a fixed standard of 5 by pressing one of two response buttons with their index fingers as fast as they could. Instructions varied within each subject: in one half of the experiment they had to press the right button for targets larger than 5 and the left button for targets smaller than 5 , while in the other half the opposite instruction was used. The order of these two sets of instructions was counterbalanced across subjects. Crucially, subjects were not told about the masked primes, but were merely informed that each trial would begin with the presentation of a 'neutral visual signal' to warn them that the target stimulus was going to appear.

\subsubsection{Stimuli}

In each trial, four visual stimuli were presented consecutively at the center of a PC screen: first, a random letter-string mask for $71 \mathrm{~ms}$ (forward mask); second, the prime which could be either the neutral symbol ' $\$$ ' or the number 1,2, 3, 4, 6, 7, 8, or 9 in Arabic or verbal notation for $43 \mathrm{~ms}$; third, a second random letter-string mask different from the first for $71 \mathrm{~ms}$; and finally, the target which could be the number 1 , 4, 6, or 9 in either Arabic or verbal notation for $200 \mathrm{~ms}$. Thus, the numbers 1, 4, 6, and 9 , which were used as targets, formed the old set primes, while the numbers 2, 3, 7, and 8, which were never used as targets, formed the new set primes (Fig. 1). Excluding the neutral trials, the design ensured that half the trials were congruent (prime and target falling on the same side of 5, and hence promoting the same response) while the other half were incongruent (prime and target falling on different sides of 5). It also guaranteed that this response congruity factor was crossed with set (old or new) as well as with the notation used for the prime and for the target. Stimuli were presented in a Triplex font, $10 \mathrm{~mm}$ high, and digits were 5-7 $\mathrm{mm}$ wide and words were $18-45 \mathrm{~mm}$ wide. The viewing distance was about $0.5 \mathrm{~m}$.
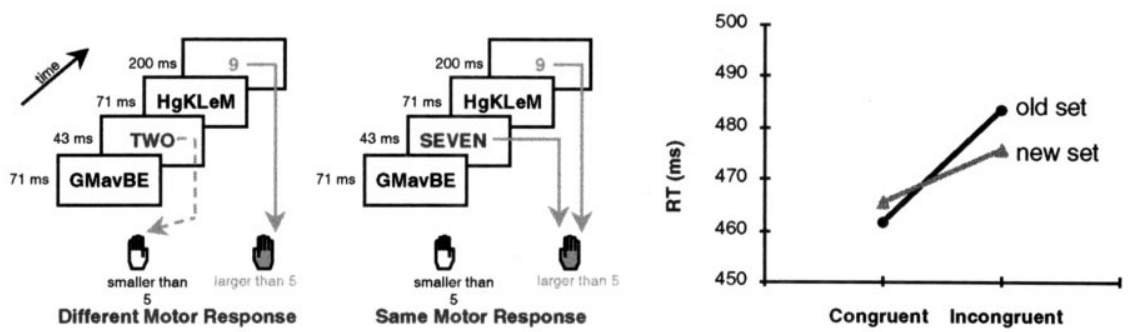

Fig. 1. (Left) Experimental paradigm. In this example, congruent and incongruent trials with new set primes are illustrated. The old set comprised the numbers 1, 4, 6, and 9, which were used both as targets and as primes. The new set comprised the numbers $2,3,7$, and 8 , which were used only as subliminal primes and never as targets. (Right) Response congruity effect in Experiment 1 . The figure shows the mean RTs (in milliseconds) for congruent trials (when both the prime and the target fell on the same side as 5) and for incongruent trials (when the prime and the target fell on opposite sides of 5), separately for old and for new prime sets. As described in the text, the congruity effect was significant in each set, and the interaction did not reach significance. 
The experiment consisted of two successive sessions of 256 experimental trials. The 512 trials corresponded to three repetitions of the 128 combinations of numerical primes and targets (Prime $(8) \times$ Prime notation $(2) \times$ Target $(4) \times$ Target notation (2)), and to 16 repetitions of the eight combinations of the target and a dollar symbol as a prime. Each session was preceded by 16 training trials. During the experimental trials, a pause appeared every 64 trials.

\subsubsection{Assessment of prime visibility}

We assessed prime visibility using both subjective and objective measures. During the number comparison experiment, any spontaneous comment of the subjects was noted. Then, immediately after the end of the comparison experiment, subjects were systematically asked several questions concerning the 'neutral signal'. Finally, at the end of the experiment, they were fully informed of the nature of the 'neutral signal' and were then asked to perform a forced-choice prime detection task designed to measure individual $d^{\prime}$ values. In this block, subjects were instructed to pay attention to the masked signal and to press the right button if a prime was present between the two masks, and the left button if it was absent. They were instructed to take any time to answer after each trial. Instructions stressed that they should press the right button whenever they had any feeling of having seen a prime in the visual sequence. The stimuli were identical to the old set trials of the main number comparison experiment. Only a single block of 64 trials was used, i.e. 32 trials at each of two prime durations (43 and $200 \mathrm{~ms}$, randomly intermixed). For each prime duration, 16 trials without the prime were mixed with 16 trials with a numerical prime $(1,4,6$, or 9, either in Arabic or in verbal notation).

\subsection{Results}

\subsubsection{Response priming}

In order to analyze response priming effects, we selected correct trials in which the prime was a number and the response time (RT) fell between 250 and $1000 \mathrm{~ms}$. We then performed a $2 \times 2 \times 2$ ANOVA on the mean RT with factors of response congruity (whether or not the prime fell on the same side of 5 as the target, and hence favored the production of the same response), prime set (old or new set) and notation change (whether the prime and the target appeared in the same notation or not). A main effect of response congruity was found $(F(1,17)=21.99, P=0.0002$, MSE $=412.28$ ) in complete concordance with our previous experiments (Dehaene et al., 1998; Koechlin, Naccache, Block, \& Dehaene, 1999). When the prime and the target fell on the same side of 5, subjects were 16 ms faster than when they fell on opposite sides of 5. Decisively, this unconscious congruity effect did not interact with the prime set factor $(F(1,17)=2.96, P=0.10$, MSE $=403.61)$. Post-hoc analyses confirmed the existence of a significant response priming effect both in the old set $(F(1,17)=13.80, P=0.002$, MSE $=609.79$, effect size $22 \mathrm{~ms})$ and, crucially, in the new set trials $(F(1,17)=8.93, P=0.008$, MSE $=206.10$, effect size $10 \mathrm{~ms}$ ) (see Fig. 2).

As in our previous experiments, the main effect of response congruity did not 


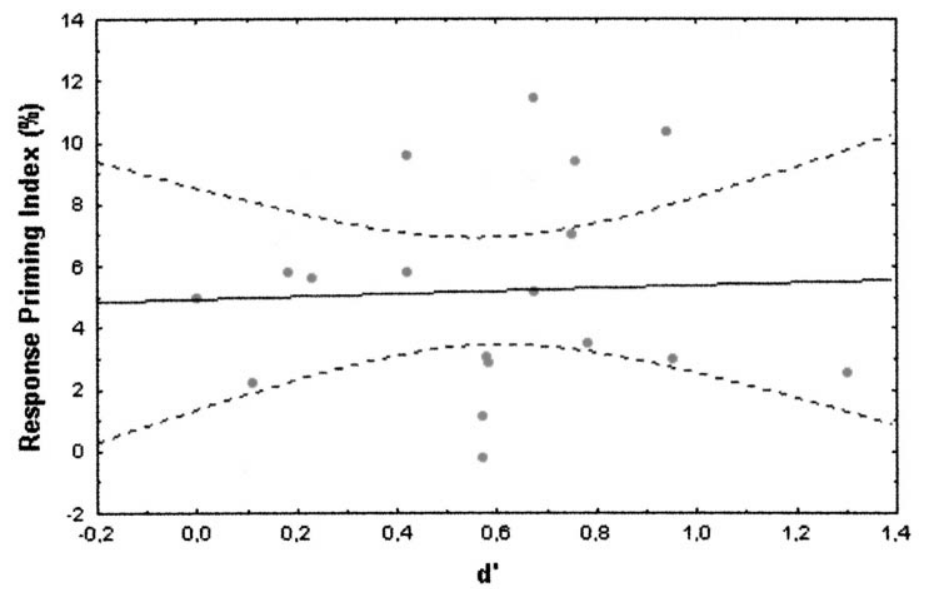

Fig. 2. Independence of response priming and $d^{\prime}$ values in Experiment 1. For each subject, a response priming index was calculated as $I=100 \times$ (mean RT for incongruent trials - mean RT for congruent trials)/(mean RT for congruent trials). This index is plotted against $d^{\prime}$ values for each subject in the forcedchoice present/absent task. Linear regression curves and corresponding $95 \%$ confidence intervals are shown. Crucially, the $y$-axis intercept was estimated at a significant value of $4.93(t(16)=2.93$, $P=0.009$ ), suggesting without consciousness (Greenwald, 1996).

interact with notation change $(F(1,17)=2.07, P=0.17, \mathrm{MSE}=403.61)$. Neither was such an interaction observed when response congruity effects were restricted either to new set or to old set primes (both $P>0.08$ ). Hence, response priming was not solely imputable to a physical repetition effect in old set prime trials. Relative to the neutral trials, we observed no significant acceleration in RT for congruent trials $(F(1,17)=1.00, P=0.33, \mathrm{MSE}=88.07)$ but a slowing down for incongruent trials $(F(1,17)=40.56, P<0.0001, \mathrm{MSE}=134.92$, effect size $19.5 \mathrm{~ms})$, again agreeing with previous results (Koechlin et al., 1999). Finally, the same ANOVAs performed on error rates did not reveal any significant effect (all $P>0.1$ ), indicating no trace of a speed-accuracy trade-off.

Further evidence for a semantic level processing of the new set primes came from an examination of the prime-target relation effect (Koechlin et al., 1999). In previous work, we found that even when focusing exclusively on congruent trials, responses were systematically faster as the numerical distance between the prime and target was smaller. For instance, responses to the conscious target 1 were systematically faster as the prime moved from 4 to 3,2 and 1. Here, the primetarget relation effect was replicated (linear contrast for the main effect of relation: $F(1,17)=12.9, P=0.002, \mathrm{MSE}=3062.94)$ and did not interact with notation change $(F<1)$. Crucially, an analysis restricted to congruent new set trials revealed a strong effect in the predicted direction. Subjects were reliably faster in responding to congruent trials in which the numerical distance between the new set prime and the target equaled 1 than to trials in which it equaled $2(F(1,17)=6.72, P=0.02$, $\mathrm{MSE}=3129.35$, effect size $17 \mathrm{~ms}$ ). This effect was independent of notation change factor (interaction $F(1,17)=0.03, P=0.86, \mathrm{MSE}=1468.29$ ) and target notation 
(both $F<0.4$ ). This effect of the semantic relation between prime and target cannot be explained by sensorimotor factors.

Analyses of learning effects also cast doubt on the DMS interpretation. According to the DMS theory, response priming should be due to the progressive automatization of the task which would progressively switch from the semantic route to a more direct sensorimotor association. An analysis of RTs as a function of training in the task did reveal a main acceleration in RT $(F(7,119)=2.43, \quad P=0.02$, MSE $=843.83$ ). However, training did not interact either with the response priming effect, or with the semantic target distance effect (slower RTs to targets close to the reference 5 than to targets far from it; both $F<1.3$ ). Both effects were significant from the first to the last block of 32 trials. Hence, there was no evidence that practicing the task resulted in increasing motor interference and decreasing semantic processing, as would be predicted by DMS.

\subsubsection{Prime visibility}

Subjective measures revealed that the primes were largely invisible. During the number comparison experiment, none of the subjects spontaneously reported having perceived the masked primes. When questioned about the 'neutral signal' immediately after the end of the experiment, eight subjects reported an impression of having seen a number in it on a few trials. Crucially, when asked to name which were these numbers, none of them reported any of the new set primes $(2,3,7$, or 8$)$ and even when explicitly asked if each of them was presented ('Did you see 2 as a prime?'), none of the 18 subjects included any of the new set primes. Moreover, the response priming effect for the new set primes was significant when restricting the analysis to the ten subjects who never reported the conscious perception of numerical primes during the questionnaire procedure $(F(1,9)=16.05, P=0.003$, MSE $=159.03)$.

Objective measures of prime visibility were obtained in the forced-choice task. For the crucial prime duration of $43 \mathrm{~ms}$, individual $d^{\prime}$ values ranged from 0.1 to 1.3 (mean 0.6, median 0.6) in 18 subjects (end-point correction procedures (Banaji \& Greenwald, 1995) were used to calculate $d^{\prime}$ in three subjects). This distribution differed significantly from a zero-centered Gaussian ( $Z$-test, $P<0.0001)$. However, this does not necessary imply conscious perception, because subjects' performance in this forced-choice task might have been contaminated by unconscious processes. Indeed, all 18 subjects claimed to have performed 'randomly' with the $43 \mathrm{~ms}$ primes. In order to circumvent this classical dilemma, Greenwald (1996) proposed an extrapolation procedure which consists of plotting individual performance in a priming task in relation to the individual $d^{\prime}$ values and using the zero intercept of this regression line as a measure of the residual priming effect for a $d^{\prime}$ of zero. Using linear regression, we found that there was no significant correlation between $d^{\prime}$ and an index of the amount of priming $(r=0.04, F(1,16)=0.03, P=0.86)$. Most crucially, the zero intercept of the regression was significantly greater than zero $(P=0.009)$ (see Fig. 2). Finally, examination of the RT distributions for congruent and incongruent trials across the 18 subjects demonstrated that the response priming effect was not due to a small proportion of trials with very slow responses, as would be expected if a small proportion of the primes had been consciously seen. 


\subsection{Discussion}

Experiment 1 provided strong evidence for semantic processing of masked numerical primes. First, the response priming was found to generalize to novel primes that were never presented as targets. Second, an effect of the semantic distance between prime and target was observed. Finally, analyses of the evolution of performance during the experiment demonstrated that subjects did not shift from a semantic to a sensorimotor response strategy with practice. Taken together, these results demonstrate that masked numbers are processed through a semantic pathway. DMS-based interpretations are clearly unable to explain such results.

Assessment of prime visibility, however, yielded less clear-cut results. While subjective measures indicated no signs of conscious perception of the masked stimuli, objective measures from a forced-choice prime detection task were significantly above chance level. We used a linear regression method introduced by Greenwald (1996) to show that priming still occurred when extrapolating to an ideal situation of chance level prime detection performance. Greenwald's method, however, may not be appropriate when the data set does not contain null or negative $d^{\prime}$ values, as is the case here. Indeed, in such a situation, the zero intercept of the regression corresponds to an extrapolation rather than to an interpolation of the data.

We speculated that the presence of positive $d^{\prime}$ values in the 'present/absent' forced-choice task could have been based on low level features of the stimuli rather than a genuine conscious perception of the primes. Indeed, prime-present trials had greater luminosity and a noticeably different flickering rhythm than prime-absent trials. At this stage, we decided to better investigate this crucial aspect of our data by replicating our main priming experiment in a new group of subjects, while measuring prime visibility using a different forced-choice task better matched with the task used in the main experiment. Subjects were simply asked to perform the comparison task on the masked prime number. If subjects are unable to consciously perceive the primes, they should be at chance on this control task.

\section{Experiment 2}

\subsection{Materials and methods}

Eighteen right-handed subjects (five males) ranging in age from 20 to 34 years (mean age 25.5 years, median age 24 years) volunteered for this study. All of them were naive as to the purpose of the experiment, and gave their informed consent. Instructions and stimuli were identical to Experiment 1 . We assessed prime visibility using both the subjective method described above (see Section 2) and a new objective measure. At the end of the main experiment, subjects were fully informed of the nature of the 'neutral signal' and were then asked to perform a forced-choice prime comparison task designed to measure individual $d^{\prime}$ values. In this block, subjects were instructed to pay attention to the masked signal and to try to compare the prime number to 5. They had to press the right button if the numerical prime was larger 
than 5, and the left button if it was smaller than 5. They were instructed to take all the time they needed before responding. The stimuli were identical to the trials used in the main number comparison experiment, except that they all contained a prime number. A single block of 64 trials, randomly selected from the list of 128 possible trials, was used.

\subsection{Results}

\subsubsection{Response priming}

We used the same statistical method as in Experiment 1 to analyze response priming effects. A main effect of response congruity was found $(F(1,17)=21.62$, $P=0.0002$, MSE $=399.26$ ) with an effect size similar to Experiment 1, congruent trials being responded to $16 \mathrm{~ms}$ faster than incongruent ones. Again, this effect did not interact with the notation change factor $(F(1,17)=1.63, P=0.22$, $\operatorname{MSE}=169.90)$ nor with prime set $(F(1,17)=0.55, P=0.47, \operatorname{MSE}=237.19)$. Crucially, post-hoc analyses replicated the existence of a reliable response congruity effect in the new set trials $(F(1,17)=10.26, P=0.0052$, MSE $=323.60$, effect size $13.6 \mathrm{~ms}$ ). Relative to neutral trials, we observed no acceleration in RT for congruent trials $(F(1,17)=0.06, P=0.81, \mathrm{MSE}=145.11)$ but a slowing down for incongruent trials $(F(1,17)=44.84, P<0.0001, \mathrm{MSE}=83.28$, effect size 16.1 ms) (see Fig. 3).

Finally, the same ANOVAs performed on error rates revealed some differences with Experiment 1. A main effect of response congruity was observed $(F(1,17)=9.45, P=0.007, \mathrm{MSE}=6.47)$, with higher error rates for incongruent trials than for congruent ones. This effect did not interact with notation change but interacted with prime set $(F(1,17)=5.27, P=0.035, \mathrm{MSE}=4.16)$. Post-hoc analyses showed that a significant effect was observed in old prime set trials $(F(1,17)=16.10, P=0.0009, \mathrm{MSE}=4.85)$ while no significant effect was observed in new prime set trials $(F<1)$.

A small but significant effect of the prime-target relation was observed

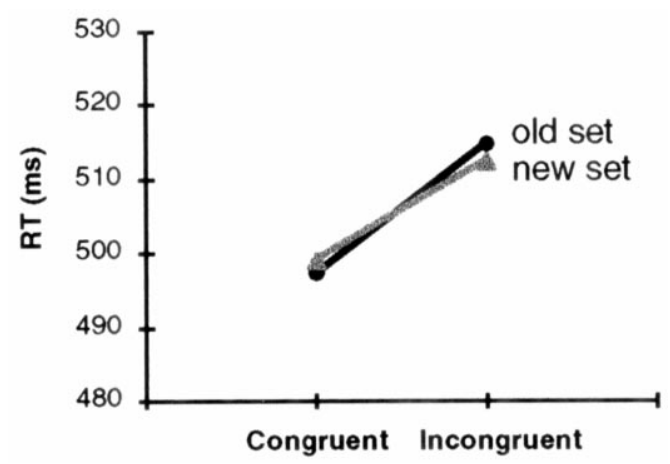

Fig. 3. Response congruity effect in Experiment 2. The figure shows the mean RTs (in milliseconds) for congruent and incongruent trials separately for old and for new prime sets. As described in the text, the congruity effect was significant in each set, and the interaction did not reach significance. 
$(F(1,17)=4.45, P=0.05, \mathrm{MSE}=1481.80)$, as there was a linear increase of RT as the numerical distance between prime and target increased from 0 to $3(509,515$, $516,518 \mathrm{~ms})$. This relation effect did not interact with notation change $(F<1)$. However, the effect was weaker than in Experiment 1 and an analysis restricted to congruent new set trials did not show a significant effect $(F<1)$.

Analyses of learning effects were very consistent with the results reported in Experiment 1. An analysis of RTs as a function of training in the task revealed a main acceleration in $\mathrm{RT}(F(7,119)=2.54, P=0.02, \mathrm{MSE}=843.19)$. However, training did not interact either with the response priming effect, or with the target distance effect (both $F<1$ ). Both effects were found to be significant as soon as the first block of 32 trials (target distance effect: $F(1,17)=41.7, P<0.0000$, MSE $=308.36$; response priming effect: $F(1,17)=3.9, P=0.03$, one-tailed, MSE $=566.86$ ). Again, there was no evidence that practicing the task resulted in increasing motor interference and decreasing semantic processing, as would be predicted by DMS.

\subsubsection{Prime visibility}

Subjective measures revealed again that the primes were largely invisible. During the number comparison experiment, none of the subjects spontaneously reported having perceived the masked primes. When questioned about the 'neutral signal' immediately after the end of the experiment, four subjects reported an impression of having seen a number in it on a few trials. Crucially, when asked to name which were these numbers, none of them reported any of the new set primes $(2,3,7$, or 8$)$ and even when explicitly asked if each of them was presented ('Did you see 2 as a prime?'), none of the 18 subjects included any of the new set primes. Moreover, the response priming effect for the new set primes was significant when restricting the analysis to the 14 subjects who never reported the conscious perception of numerical primes during the questionnaire procedure $(F(1,13)=6.21, \quad P=0.03$, MSE $=387.41$, effect size $13.1 \mathrm{~ms}$ ).

Objective measures of prime visibility were obtained in the prime comparison task. Individual $d^{\prime}$ values ranged from -0.57 to +0.65 (mean +0.01 , median $-0.04)$. This distribution did not differ significantly from a zero-centered Gaussian (Z-test, $P=0.42) .{ }^{1}$ We again performed Greenwald's analysis (Greenwald, 1996) to obtain an interpolated value of priming for null $d^{\prime}$ in our population of subjects. Using linear regression, we found a non-significant correlation between $d^{\prime}$ and an

\footnotetext{
${ }^{1}$ An anonymous referee suggested that interference induced by the presence of a visible target immediately following the prime might have led to an underestimation of prime perceptibility. Indeed, because our design orthogonally crosses primes and targets, if subjects' prime comparison responses were heavily influenced by the presence of a target, they could not possibly give any indication about prime perceptibility. In fact, however, subjects' responses were independent of the target-induced response (the mean ratio of correct responses according to the target was 50\%, SD 9\%, $Z=0, P=0.5$ ). Furthermore, even on the $50 \%$ of trials where subjects responded on the side opposite to that induced by the target, subjects' prime comparison performance remained at chance level (mean $49 \%$ correct, SD $8 \%, Z=-0.71$, $P=0.8$ ). In conclusion, it seems that the presence of the target number did not influence subjects' performance in the prime comparison task.
} 


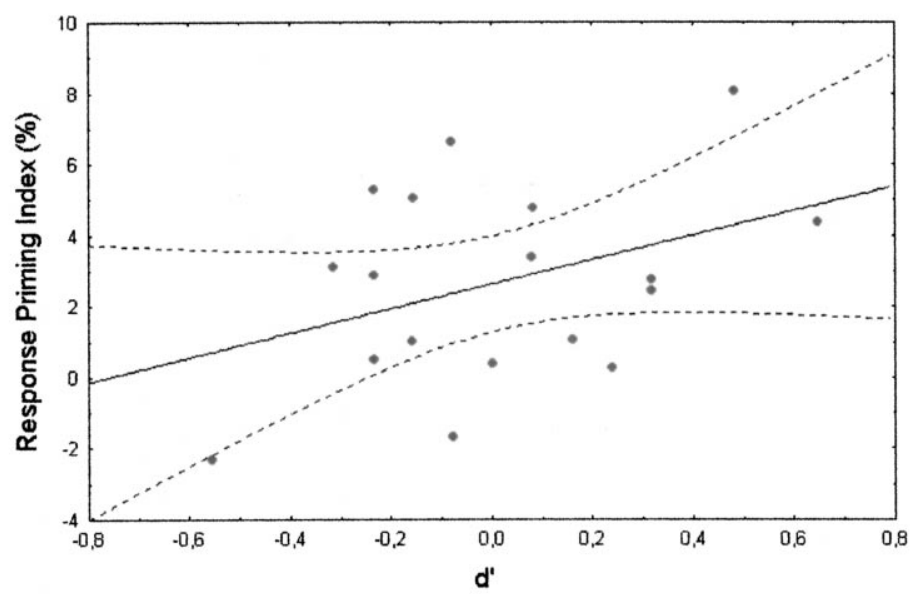

Fig. 4. Response priming indices in Experiment 2. The index is plotted against $d^{\prime}$ values for each subject in the forced-choice prime comparison task. Linear regression curves and corresponding $95 \%$ confidence intervals are shown. Crucially, the $y$-axis intercept was estimated at a significant value of 2.63 $(t(16)=4.22, P=0.0006)$, indicating priming without consciousness (Greenwald, 1996).

index of the amount of priming $(r=0.38, F(1,16)=2.69, P=0.12)$. Most crucially, the constant term of the regression was significantly greater than zero $(P=0.0006)$ (see Fig. 4).

\subsection{Discussion}

The aim of Experiment 2 was to replicate Experiment 1 with a better measure of the degree of conscious perception of numerical primes. Analysis of priming effects closely replicated the major findings of Experiment 1. A strong response priming effect was observed and this effect remained significant for the new prime trials, in which subjects processed primes that were never presented as targets. As in Experiment 1 , response priming effects did not interact with notation factors. The numerical relation between prime and target also had a linear effect on RTs, although in Experiment 2 this was significant overall, but not when restricting the analysis only to new set trials.

Finally, learning analysis replicated our results from Experiment 1, and in particular the finding of a response priming effect from the very first block onwards.

More important is the prime visibility measure which motivated this experiment. Using an explicit prime comparison task we found that subjects were at chance level. The prime detection task used in Experiment 1 could have been performed using low level visual features of the stimuli, such as a difference in luminance on primeabsent versus prime-present trials. When such a strategy was prevented in Experiment 2, performance dropped to chance, indicating that the primes were invisible.

In conclusion, Experiment 2 enabled us to replicate semantic priming effects, 
similar to those found in Experiment 1, and to show that subjects did not consciously perceive the numerical primes.

\section{General discussion}

The aim of the present study was to test two alternative interpretations of the semantic response interference effects with masked primes. One interpretation is that those effects stem from an unconscious categorization of primes at a semantic level. Another proposes that they are caused by direct motor specification via a nonsemantic stimulus-response chain. The results were clear-cut in supporting the semantic hypothesis.

First, we observed a significant response priming effect for prime stimuli that were never presented as targets and for whom no stimulus-response chains could therefore have been acquired. It should be noted that the existence of a trend of an interaction between response priming and prime set (stronger response priming for old set primes than for new set primes) does not weaken our semantic interpretation. The critical point is to observe a reliable response priming effect for new set primes which demonstrates the existence of a semantic processing of prime numbers. The trend towards a stronger response priming effect for old set primes could reflect the existence of both semantic and associative streams of processing. Alternatively, it might also reflect an attentional or training set effect for the consciously seen quantities at the semantic level. Thus, even if this interaction became significant when testing more subjects, it would not be diagnostic of the models tested here.

Second, we found faster responses when the prime and target were numerically close than when they were further apart. This effect reflects the metric of semantic proximity between numerical quantities and cannot be easily reconciled with a DMS-based model. Note that both effects (response priming and prime-target relation) were independent of the notation used for displaying the prime and target (Arabic digit or spelled-out words), thus confirming that they arise from a notation-independent semantic level. In two previous studies (Dehaene et al., 1998; Koechlin et al., 1999) we also found response priming effects independent from notation factors. The only discrepancy between the present data and our past results concerns relation effects (faster responses when the prime and target are numerically close than when they are further apart). We previously found significant relation effects only for trials in which the prime and the target were presented in the same notation (Koechlin et al., 1999), whereas we found here a relation effect unaffected by notation change. One possibility, which would require further investigation, is that subtle differences in the experimental paradigm, such as the longer prime duration $(66 \mathrm{~ms})$ used by Koechlin et al., were somehow responsible for the lack of cross-notation transfer of the relation effect. Perhaps a more plausible possibility, however, is that this was a spurious effect. Indeed, the relation $\times$ notation change $\times$ target notation interaction reported by Koechlin et al. was quite weak $(P=0.051)$. All other indices of numerical priming that we have obtained are totally independent 
of number notations, suggesting that cross-notation semantic priming is the norm rather than the exception.

Third, an analysis of training effects revealed that the unconscious response priming effect was already present within the first block of 32 trials and that its intensity did not increase with time, which speaks against the hypothesis of a sensorimotor chain progressively building strength through associative learning. Along the same lines, we found no reduction of the semantic distance effect with training, and hence no trace of a progressive attenuation of the conscious semantic process of targets, as would be predicted by the hypothesis of a progressive transfer from a semantic to a non-semantic processing route.

Can we precisely estimate the level of conscious perception of the primes? In Experiment 1, while subjective measures of prime awareness suggested that none of our 18 subjects consciously perceived the primes during the main experiment, objective measures showed a small but reliable above-chance performance in a prime detection task. This discrepancy between subjective and objective measures deserves several remarks. First, the task we used to objectively evaluate prime visibility is very different from the main task during which subjective measures and priming effects were obtained. In the forced-choice detection task, subjects focused their attention on the primes and could perform the requested present/absent judgment on the basis of 'low level' visual features. By contrast, in the main experiment, subjects focused their attention on the target stimuli and priming effects revealed access to a semantic representation. Second, slight above-chance performance in a forced-choice task can reflect contamination by unconscious processes rather than explicit conscious performance. Indeed, after the forced-choice task, all subjects reported the subjective impression of having responded at chance during the whole block of trials. Third, $d^{\prime}$ values and the amount of response priming were not correlated, and we found a significantly above-chance priming effect when the results were extrapolated to a theoretical point where $d^{\prime}=0$. In brief, one may wonder whether subjects' subjective reports of not seeing the primes were not a more reliable measure of prime awareness than the objective $d^{\prime}$ measure which was obtained in a distinct block and task. Given the crucial importance of this issue to our semantic priming results, we decided in Experiment 2 to investigate prime awareness further by using a forced-choice task of prime comparison better matched with the main task. In this task, subjects performed at chance level, while priming effects were replicated. This study provides strong evidence for the unconscious nature of our semantic priming effects.

Why do we find support for a semantic stage in subliminal number processing, while Abrams and Greenwald (in press) found only evidence for a non-semantic association between fragments of the visual primes and motor responses? Several differences might be critical. First, Abrams and Greenwald used a complex task that required positive or negative valence judgment. It seems unlikely that access to valence information is computed in a highly automated manner. On the contrary, there are many indications that access to the quantity meaning of numbers is a highly automated process that cannot be prevented, even in situations where it is totally irrelevant (Brysbaert, 1995; Dehaene \& Akhavein, 1995; Dehaene, Bossini, \& 
Giraux, 1993; Fias, Brysbaert, Geypens, \& d'Ydewall, 1996; LeFevre, Bisanz, \& Mrkonjic, 1988). Second, the experimental conditions used by Abrams and Greenwald forced subjects to answer in a very narrow time response window following the stimulus. This may have encouraged subjects to rely on a non-semantic fragmentbased process, which was indeed possible because the same target words were repeatedly used throughout the experiment. Similar factors may have contributed to the failure by Damian (in press) to observe generalization to novel subliminal primes in a word categorization task. However, our finding is not unique in that Dell'Acqua and Grainger (1999) also report evidence of unconscious access to meaning of pictures using a masked priming paradigm where primes were never presented as targets (Experiment 1A).

In conclusion, the present work does not deny that direct sensorimotor chains may underlie some subliminal priming experiments. However, it demonstrates that authentic instances of unconscious semantic manipulation exist. In the future, neuroimaging techniques could be used to directly image subliminal semantic activations. Luck et al. (1996) already demonstrated that a classical electrophysiological correlate of semantic processing, the N400 component, is evoked in exactly the same way by conscious words and unconscious words presented during the 'attentional blink'. Likewise, we have observed that subliminal number primes modulate fMRI activation in parietal areas known to be involved in semantic quantity processing (Naccache \& Dehaene, 2001). Such data provide an even stronger empirical basis for unconscious semantic processing.

\section{References}

Abrams, R. L., \& Greenwald, A. G. (in press). Parts and wholes in unconscious priming.

Banaji, M. R., \& Greenwald, A. G. (1995). Implicit gender stereotyping in judgments of fame. Journal of Personality and Social Psychology, 68, 181-198.

Brysbaert, M. (1995). Arabic number reading: on the nature of the numerical scale and the origin of phonological recoding. Journal of Experimental Psychology: General, 124, 434-452.

Damian, M. F. (in press). Congruity effects evoked by subliminally presented primes: automaticity rather than semantic processing. Journal of Experimental Psychology: Human Perception and Performance.

Dehaene, S., \& Akhavein, R. (1995). Attention, automaticity, and levels of representation in number processing. Journal of Experimental Psychology: Learning, Memory, and Cognition, 21 (2), 314-326.

Dehaene, S., Bossini, S., \& Giraux, P. (1993). The mental representation of parity and number magnitude. Journal of Experimental Psychology: General, 122 (3), 371-396.

Dehaene, S., Naccache, L., Le Clec, H. G., Koechlin, E., Mueller, M., Dehaene-Lambertz, G., van de Moortele, P. F., \& Le Bihan, D. (1998). Imaging unconscious semantic priming. Nature, 395 (6702), 597-600.

Dell'Acqua, R., \& Grainger, J. (1999). Unconscious semantic priming from pictures. Cognition, 73 (1), B1-B15.

Draine, S. C., \& Greenwald, A. G. (1998). Replicable unconscious semantic priming (see comments). Journal of Experimental Psychology: General, 127 (3), 286-303.

Eimer, M., \& Schlaghecken, F. (1998). Effects of masked stimuli on motor activation: behavioral and electrophysiological evidence. Journal of Experimental Psychology: Human Perception and Performance, 24 (6), 1737-1747.

Ferrand, L., Grainger, J., \& Segui, J. (1994). A study of masked form priming in picture and word naming. Memory and Cognition, 22 (4), 431-441. 
Fias, W., Brysbaert, M., Geypens, F., \& d'Ydewall, G. (1996). The importance of magnitude information in numerical processing: evidence from the SNARC effect. Mathematical Cognition, 2, 95-110.

Forster, K. I. (1999). The microgenesis of priming effects in lexical access. Brain and Language, 68 (1-2), $5-15$.

Greenwald, A. G. (1996). Three cognitive markers of unconscious semantic activation. Science, 273 (5282), 1699-1702.

Koechlin, E., Naccache, L., Block, E., \& Dehaene, S. (1999). Primed numbers: exploring the modularity of numerical representations with masked and unmasked semantic priming. Journal of Experimental Psychology: Human Perception and Performance, 25 (6), 1882-1905.

LeFevre, J., Bisanz, J., \& Mrkonjic, L. (1988). Cognitive arithmetic: evidence for obligatory activation of arithmetic facts. Memory and Cognition, 16, 251-267.

Luck, S. J., Vogel, E. K., \& Shapiro, K. L. (1996). Word meanings can be accessed but not reported during the attentional blink. Nature, 383 (6601), 616-618.

Marcel, A. J. (1983). Conscious and unconscious perception: experiments on visual masking and word recognition. Cognitive Psychology, 15 (2), 197-237.

Naccache, L., \& Dehaene, S. (2001). The priming method: imaging unconscious repetition priming reveals an abstract representation of number in the parietal lobels. Manuscript submitted for publication.

Neumann, O., \& Klotz, W. (1994). Motor responses to nonreportable, masked stimuli: where is the limit of direct parameter specification? In C. Umiltà, \& M. Moscovitch, (Eds.), Conscious and nonconscious information processingAttention and performance (Vol. XV. pp. 123-150). Cambridge, MA: MIT Press.

Whalen, P. J., Rauch, S. L., Etcoff, N. L., McInerney, S. C., Lee, M. B., \& Jenike, M. A. (1998). Masked presentations of emotional facial expressions modulate amygdala activity without explicit knowledge. Journal of Neuroscience, 18 (1), 411-418. 